\title{
Cost-Effective Computational Method for Radiation Heat Transfer in Semi-Crystalline Polymers
}

\author{
Sinan Boztepe $e^{1, \mathrm{a}}$, Rémi Gilblas ${ }^{1, \mathrm{~b}}$, Olivier de Almeida ${ }^{1, \mathrm{c}}$, Yannick Le Maoult ${ }^{1, \mathrm{~d}}$ and \\ Fabrice Schmidt ${ }^{1, \mathrm{e}}$ \\ ${ }^{1} 1$ Université de Toulouse ; IMT Mines Albi, ICA (Institut Clément Ader) ; Campus Jarlard, F-81013 \\ Albi cedex 09, France. \\ ${ }^{a)}$ sboztepe@mines-albi.fr ${ }^{\text {b) }}$ rgilblas@mines-albi.fr ${ }^{c)}$ olivier.dealmeida@mines-albi.fr ${ }^{\mathrm{d})}$ lemaoult@mines-albi.fr \\ e) fabrice.schmidt@mines-albi.fr
}

\begin{abstract}
This paper introduces a cost-effective numerical model for infrared (IR) heating of semi-crystalline polymers. For the numerical and experimental studies presented here semi-crystalline polyethylene (PE) was used. The optical properties of PE were experimentally analyzed under varying temperature and the obtained results were used as input in the numerical studies. The model was built based on optically homogeneous medium assumption whereas the strong variation in the thermo-optical properties of semi-crystalline PE under heating was taken into account. Thus, the change in the amount radiative energy absorbed by the PE medium was introduced in the model induced by its temperaturedependent thermo-optical properties. The computational study was carried out considering an iterative closed-loop computation, where the absorbed radiation was computed using an in-house developed radiation heat transfer algorithm RAYHEAT- and the computed results was transferred into the commercial software -COMSOL Multiphysics- for solving transient heat transfer problem to predict temperature field. The predicted temperature field was used to iterate the thermo-optical properties of PE that varies under heating. In order to analyze the accuracy of the numerical model experimental analyses were carried out performing IR-thermographic measurements during the heating of the PE plate. The applicability of the model in terms of computational cost, number of numerical input and accuracy was highlighted.
\end{abstract}

\section{INTRODUCTION}

In terms of modeling the radiation heat transfer in optically heterogeneous media, numerous examples of modelling approaches exist in literature that adopt well-known optical scattering theories, such as Rayleigh-DebyeGans [1] or Mie [2,3] theory which allows to predict the spatial distribution of the scattered light intensity accurately. However, such radiation heat transfer models that handle complex radiation physics may be computationally costly, especially if spectral or geometrical complexity in a heated component is involved [4]. In contrast to laser heating applications, the radiation emitted by an IR source and transferred through a polymer medium is not monochromatic, but in a range, which introduces such a complexity in terms of modeling of optical scattering, as the scattering behavior may change under varying wavelength in the spectral range of emitted radiation. Such a wavelength-dependent scattering behavior in semi-crystalline polymers was discussed in $[5,6]$.

Apart from the computational cost due to spectral complexity of IR radiation, the strong coupling between the microcrystalline structure of unfilled semi-crystalline thermoplastics and their optical scattering behavior may also increase the complexity of a numerical approach, and thus computational cost. It is known that optical scattering in semi-crystalline polymers are strongly related to their microcrystalline structure where the optical scattering behavior may evolve under heating, due to a potential change in their microcrystalline phase at the temperatures close to their melting ranges. It may therefore be useful to adopt a numerical model that considers this temperaturedependent optical behavior. Potente et al [7] proposed temperature-dependent optical properties for semi-crystalline polypropylene (PP), named as "effective absorption constant". However the temperature dependency in absorption characteristics of PP was modeled based on the change in the density of the polymer in the corresponding temperature range, so that it lacks the physical background due to no experimental characterization on its optical properties was performed. Similarly, temperature-dependent absorption properties were introduced as a numerical 
input for laser transmission welding (LTW) process simulations of short glass fiber filled and unfilled PP and PA6 in [8] whereas the behavior about the absorption characteristics under varying temperature was not highlighted.

In this paper, a cost-effective numerical approach was proposed for unfilled semi-crystalline thermoplastics considering the temperature-dependent behavior in their optical characteristics. For the study, temperaturedependent directional-hemispherical transmittance $\left(T_{\lambda}{ }^{i, \cap}(T)\right)$ and reflectance $\left(R_{\lambda}{ }^{i, \cap}(T)\right)$ of semi-crystalline PE was experimentally analyzed considering different temperatures between $25^{\circ} \mathrm{C}$ and $128^{\circ} \mathrm{C}$. Assuming an optically homogeneous medium, the measured $T_{\lambda}{ }^{i, \cap}(T)$ and $R_{\lambda}{ }^{i, \cap}(T)$ were used to calculate the temperature-dependent extinction coefficient $\left(\beta_{\lambda}(\mathrm{T})\right.$ ), which considers an evolution in the optical characteristics of PE under heating condition. The calculated $\beta_{\lambda}(\mathrm{T})$ were then used as numerical input so that the temperature-dependency of absorbed radiative energy by the PE medium was taken into account in the model. The computational study was carried out considering an iterative closed-loop computation, using an in-house developed radiation heat transfer algorithm RAYHEAT- and the commercial software -COMSOL Multiphysics- where the closed-loop iterations was established using MATLAB LiveLink. In order to analyze the accuracy of the numerical model experimental analyses were carried out performing IR-thermographic measurements for the case of IR-heating of the PE plate. The model developed here proposes a numerical approach to consider the optical scattering and its effect under heating condition indirectly that is governed by microcrystalline morphology. In other words, the effect of optical scattering on the optical characteristics of semi-crystalline PE was taken into account without modeling the spatial distribution of the scattered light intensity which offers computationally cost-effective numerical solutions.

\section{TEMPERATURE DEPENDENCY IN THE THERMO-OPTICAL PROPERTIES OF SEMI-CRYSTALLINE POLYMERS}

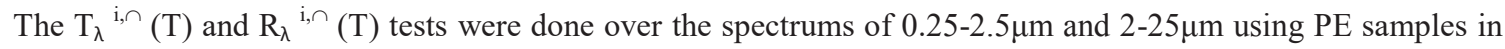
three different thicknesses. All the samples were quenched into liquid nitrogen $\left(\mathrm{LN}_{2}\right)$. The details of the sample preparation is presented in [5]. $T_{\lambda}{ }^{i, \cap}(T)$ and $R_{\lambda}{ }^{i, \cap}(T)$ of each PE sample were measured at various temperature steps, between $25^{\circ} \mathrm{C}$ and $128^{\circ} \mathrm{C}$. The $\mathrm{T}_{\lambda}{ }^{\mathrm{i}, \cap}(\mathrm{T})$ tests were performed employing FT-IR spectrometer (Perkin Elmer 950) combined with an integrating sphere. For the tests, a heating stage (Bruker A599) was positioned at the entrance port of the integrating sphere, so that the samples were mounted into the heating stage and their $T_{\lambda}{ }^{i, \cap}(T)$ were measured. For the measurement at each step, the samples were heated up and waited for 2 minutes in order to reach a steady-state temperature. In total, $\mathrm{T}_{\lambda}{ }^{\mathrm{i}, \cap}(\mathrm{T})$ of each PE sample was measured at 14 different temperature points. Similarly, for each PE sample, $R_{\lambda}{ }^{i, \cap}(T)$ measurements were performed at 10 different temperature steps. The $\mathrm{R}_{\lambda}{ }^{\mathrm{i}, \cap}(\mathrm{T})$ tests were done employing SOC-100 model Hemispherical Directional Reflectometer (HDR) built by Surface Optics Corporation. For these tests, the samples were heated using the heating unit of the SOC-100 HDR. Details of the PE samples and the temperature steps of the tests are presented in Table 1.

TABLE 1. The PE samples and the testing characteristics of the $T_{\lambda}{ }^{i, \cap}(T)$ and $R_{\lambda}{ }^{i, n}(T)$ tests

\begin{tabular}{ccc}
\hline Type of measurement & Sample thickness $(\mathbf{m m})$ & $\begin{array}{c}\text { Temperature steps for } \\
\text { measurements }\left({ }^{\mathbf{0}} \mathbf{C}\right)\end{array}$ \\
\hline $\mathrm{T}_{\lambda}{ }^{\mathrm{i}, \cap}(\mathrm{T})$ & $0.25,0.50$ and 0.75 & $25,48,71,84,97,110,114,116$, \\
& & $118,120,121,122,123,126,128$ \\
$\mathrm{R}_{\lambda}{ }^{\mathrm{i}, \cap}(\mathrm{T})$ & $0.25,0.50$ and 0.75 & $25,71,97,103,110,114,118$, \\
\end{tabular}

It should be noted that the temperature steps mentioned in Table 1 represent the actual temperature on the sample surface where the measurements were obtained. In other words, it was observed that the set temperature on the heating devices that were used in $\mathrm{T}_{\lambda}{ }^{\mathrm{i}, \cap}(\mathrm{T})$ and $\mathrm{R}_{\lambda}{ }^{\mathrm{i}, \cap}(\mathrm{T})$ tests and the actual temperature on the measurement zone are different. Therefore, preliminary tests were done in order to determine the actual temperature on the measurement zone. The actual temperatures adopted in the $\mathrm{R}_{\lambda}{ }^{\mathrm{i}, \cap}(\mathrm{T})$ tests were determined using identically thick dummy PE samples on the heating unit of SOC-100 HDR. In addition, the details of the preliminary tests for the transmittance measurements is revealed in [9]. In Fig. 1(a) and (b), the typical trend obtained in each $\mathrm{T}_{\lambda}{ }^{\mathrm{i}, \cap}(\mathrm{T})$ and $\mathrm{R}_{\lambda}{ }^{\mathrm{i}, \cap}(\mathrm{T})$ tests were presented respectively. It is seen in Fig. 1(a) that the $\mathrm{T}_{\lambda}{ }^{\mathrm{i}, \cap}$ of the $0.75 \mathrm{~mm}$ thick sample increases under heating where the increase is more pronounced after $116{ }^{\circ} \mathrm{C}$, that reflects the temperatures close to 
the melting range of the polymer [5]. It should also be mentioned that no significant change was observed in $\mathrm{T}_{\lambda}{ }^{\mathrm{i}, \cap}$ for the wavelengths greater than $1.7 \mu \mathrm{m}$, where also a significant noise was detected during the measurements at high temperatures. It was thus assumed that $\mathrm{T}_{\lambda}{ }^{\mathrm{i}, \cap}$ remains unchanged under heating considering the spectrum between 1.7 and $2.5 \mu \mathrm{m}$. In contrast to the trend in $\mathrm{T}_{\lambda}{ }^{\mathrm{i}, \cap}(\mathrm{T})$, there is an inverse relation between the $\mathrm{R}_{\lambda}{ }^{\mathrm{i}, \cap}$ of $\mathrm{PE}$ and temperature. As shown in Fig. 1(b), the temperature change is less effective on the reflectance properties of PE in comparison to its temperature-dependent transmittance. For the sake of clarity in the trends observed at both the $\mathrm{T}_{\lambda}$ $\mathrm{i}, \cap(\mathrm{T})$ and $\mathrm{R}_{\lambda}{ }^{\mathrm{i}, \cap}(\mathrm{T})$, certain number of data are presented in Fig. 1(a) and (b).

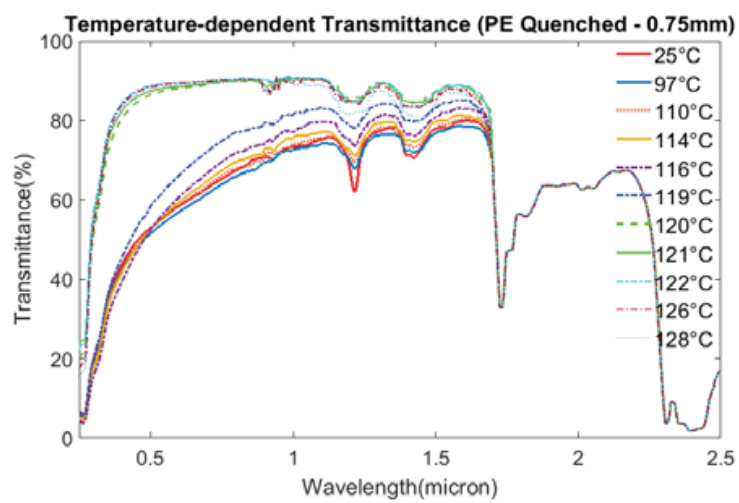

(a)

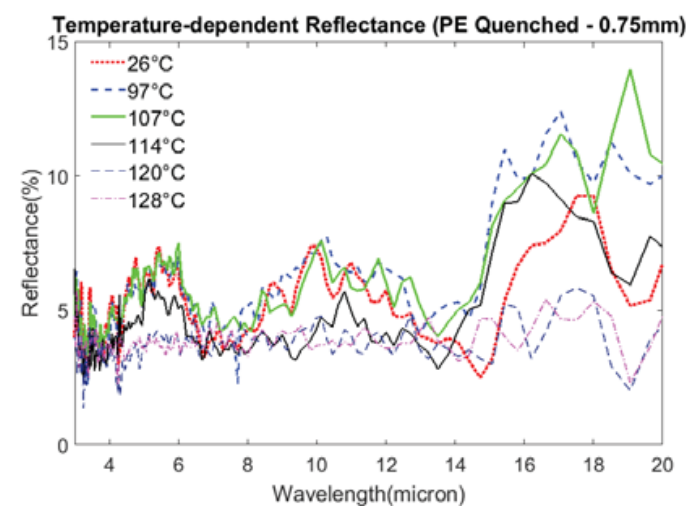

(b)

FIGURE 1. Typical trend obtained in $\mathrm{T}_{\lambda}{ }^{\mathrm{i}, \cap} \mathrm{R}_{\lambda}{ }^{\mathrm{i}, \cap}$ measurements of PE polymer under varying temperature

As our previous studies [5,10] showed that the strong coupling between microcrystalline morphology and the transmittance characteristics of PE becomes negligible in MIR range $(2-25 \mu \mathrm{m})$, no temperature dependency was assumed for transmittance characteristics of PE in MIR range. Hence, $\mathrm{T}_{\lambda}{ }_{\mathrm{i}, \curvearrowright}$ of the identical PE samples measured at room temperature was adopted for this range. Conversely, no temperature-dependent measurements were able to be obtained for the reflectance measurement considering the wavelengths lower than $2 \mu \mathrm{m}$, due to the limitations in the testing devices employed and the spectral responses of their detectors. It was therefore assumed that reflectance characteristics of $\mathrm{PE}$ are independent of temperature. Although this is not a rigorous assumption, further analyses on the PE samples prepared with different microcrystalline structure were tested at room temperature and a slight effect of the microcrystalline morphology on the reflectance was observed. It may therefore be stated that the reflectance behavior of PE may not change dramatically in case that a change in its microcrystalline structure under varying temperature is seen. Based on the measured $T_{\lambda}{ }^{i, \cap}(T), R_{\lambda}{ }^{i, \cap}(T)$ and the adopted assumptions, $\beta_{\lambda}(T)$ of PE was calculated considering an optically homogeneous medium. Using the transmittance and the reflectance characteristics of an optically homogeneous medium, it may be possible to define the $\beta_{\lambda}$ [11]. Hence, $\beta_{\lambda}$ may be calculated for each adopted temperature step that eventually leads to:

$$
\begin{gathered}
\mathrm{A}_{\lambda}{ }^{\mathrm{i}, \cap}(\mathrm{T})+\mathrm{T}_{\lambda}{ }^{\mathrm{i}, \cap}(\mathrm{T})+\mathrm{R}_{\lambda}{ }^{\mathrm{i}, \cap}(\mathrm{T})=1 \text { with; } \\
T_{\lambda}{ }^{\mathrm{i}, \cap}(T)=\frac{\left(1-\rho_{\lambda}\right)^{2} \tau_{\lambda}}{1-\left(\rho_{\lambda} \tau_{\lambda}\right)^{2}} \quad \text { and } R_{\lambda}^{\mathrm{i}, \cap}(T)=\rho_{\lambda}\left[1+\tau_{\lambda}{ }^{2} \frac{\left(1-\rho_{\lambda}\right)^{2} \tau_{\lambda}{ }^{2}}{1-\left(\rho_{\lambda} \tau_{\lambda}\right)^{2}}\right]
\end{gathered}
$$

The computation of $\beta_{\lambda}$ at each temperature step was carried out in MATLAB, adopting an inverse method based on SQP (Sequential Quadratic Programming) estimation algorithm, so that the equation 2 was solved for computing $\rho_{\lambda}$ and $\tau_{\lambda}$ values, and eventually $\beta_{\lambda}$. The background of this in-house developed estimation algorithm is explained further in [12]. In Fig. 2, the computed $\beta_{\lambda}(\mathrm{T})$ values are presented considering the spectrum between 0.6-7 $\mu \mathrm{m}$. Again, for the sake of clarity, some of the computed values are not presented in the figure. Furthermore, the drop in $\beta_{\lambda}$ under heating is illustrated further in the figure at right, including the spectrum between $0.6-1.6 \mu \mathrm{m}$. This may be plausible, as the change in the $\mathrm{T}_{\lambda}{ }^{\mathrm{i}, \cap}(\mathrm{T})$ values is significant in this spectrum. Apart from this, it is clear that the trend in $\beta_{\lambda}(\mathrm{T})$ is monotonically decreasing under heating, except the computed $\beta_{\lambda}$ at $128{ }^{\circ} \mathrm{C}$. This is due to the fact that a slight drop in $\mathrm{T}_{\lambda}{ }^{\mathrm{i}, \cap}$ were observed for all the PE samples after the measurement at $126{ }^{\circ} \mathrm{C}$, which resulted to have slightly higher $\beta_{\lambda}$ computed at $128^{\circ} \mathrm{C}$, in comparison to the computed one at $126{ }^{\circ} \mathrm{C}$. This behavior was also observed in our previous study [9], which can be attributed to disintegration of polymer at semi-molten state, and therefore potential thickening in the measurement zone of the vertically positioned sample. Because of this limitation in $\mathrm{T}_{\lambda}{ }^{\mathrm{i}, \cap}(\mathrm{T})$ tests, the calculated extinction coefficient at $128{ }^{\circ} \mathrm{C}$ is ignored in this study. Hence the $\beta_{\lambda}(\mathrm{T})$ computed at 13 temperature steps were used as input in the numerical model. 


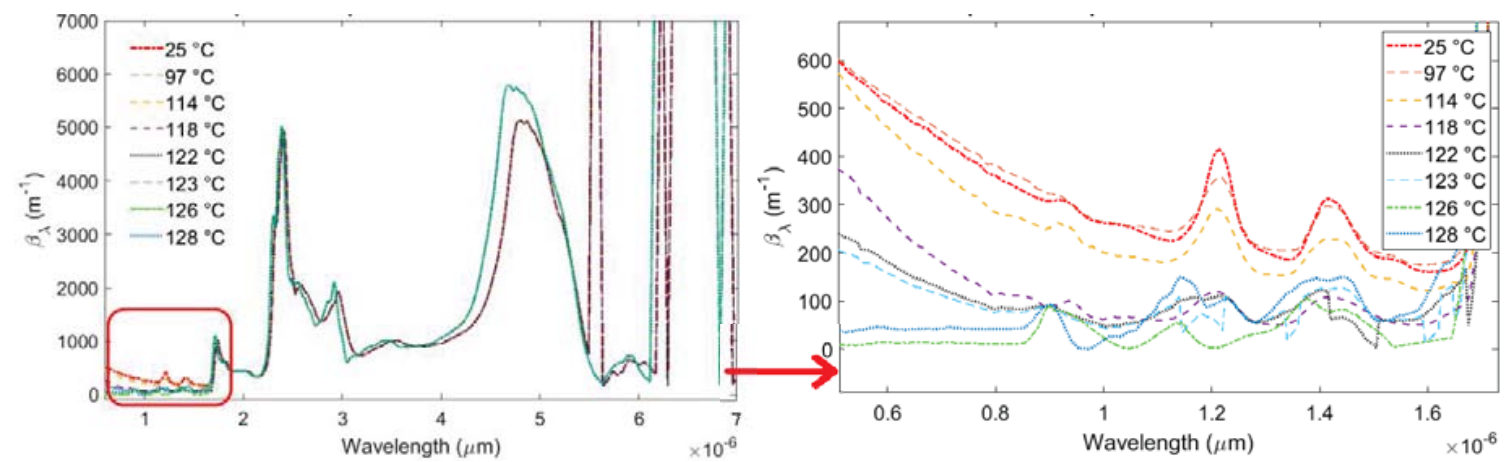

FIGURE 2. $\beta_{\lambda}$ (T) of PE polymer between room temperature and $128^{\circ} \mathrm{C}$

\section{RADIATION HEAT TRANSFER MODELING APPROACH WITH TEMPERATURE- DEPENDENT OPTICAL PROPERTIES AND EXPERIMENTAL COMPARISONS}

\section{Background of the numerical model}

As aforementioned, the computational study was carried out considering an iterative closed-loop computation, where the absorbed radiation was computed using in-house developed algorithm, RAYHEAT, and transferred into COMSOL. The current version of RAYHEAT is based on optically homogeneous medium assumption, hence, attenuation of radiative energy is computed adopting Beer-Lambert Law, as shown in Equation 3 [11]. The further details about RAYHEAT, including ray tracing method, and discretization of emitted rays can be found in [13].

$$
\frac{d I_{\lambda}}{d z}=-\beta_{\lambda}(z) I_{\lambda}(z)=-\left(\kappa_{\lambda}(z)+D_{\lambda}(z)\right) I_{\lambda}(z)
$$

The absorbed energy, namely radiative source term $\left(\nabla \cdot q_{\mathrm{r}}\right)$, at the depth " $\mathrm{z}$ " of a heated medium can be defined in Equation 4 , at which $\beta_{\lambda}$ is the sum of both absorption $\left(\kappa_{\lambda}\right)$ and scattering coefficients $\left(D_{\lambda}\right)$ :

$$
\nabla \cdot q_{r}(z)=\int_{0}^{\infty}-\beta_{\lambda} I_{\lambda}(0) e^{-\beta_{\lambda^{z}}} d \lambda=\int_{0}^{\infty}-\left(\kappa_{\lambda}+D_{\lambda}\right) I_{\lambda}(0) e^{-\left(\kappa_{\lambda}+D_{\lambda}\right) z} d \lambda
$$

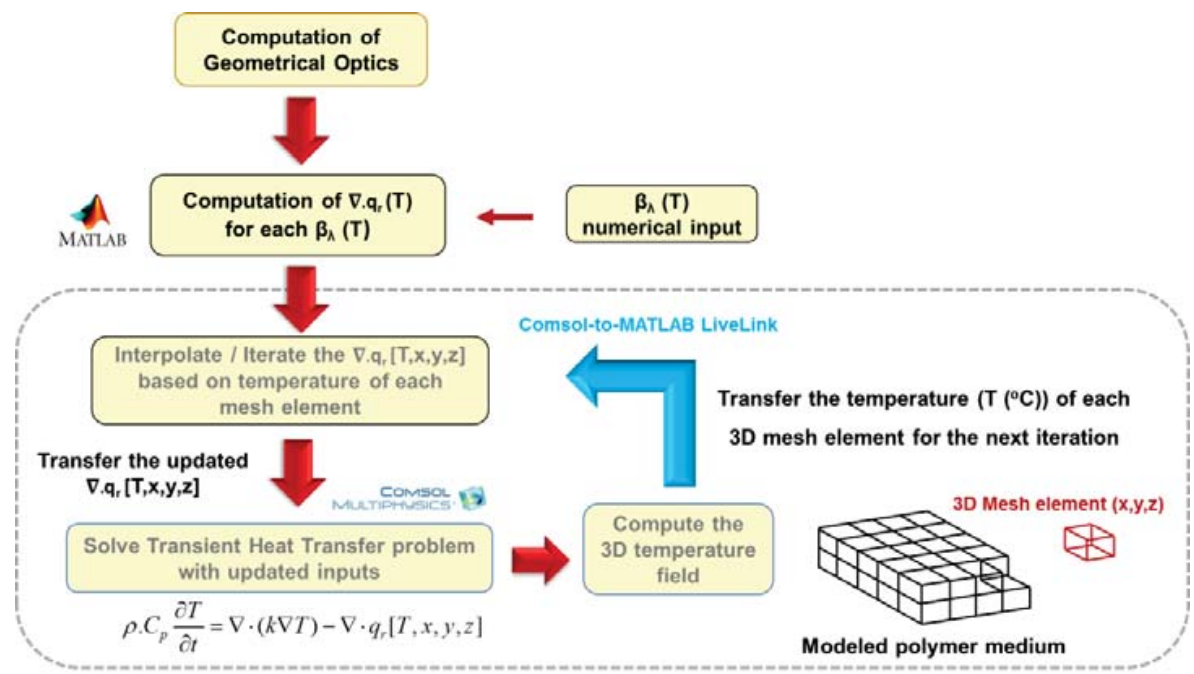

FIGURE 3. Working algorithm of the developed numerical model

In order to take into account $\beta_{\lambda}(\mathrm{T})$ as a numerical input in the model, a close-loop iterative approach was developed. The working algorithm of the model is displayed schematically in Fig. 3. As RAYHEAT is a MATLABbased code, the iterative closed-loop between RAYHEAT and COMSOL was established using MATLAB LiveLink feature which allows to transfer the computed $\nabla . q_{\mathrm{r}}$ into COMSOL as a numerical input, solves the transient heat 
transfer problem to predict the temperature field and calls back RAYHEAT for updating the $\nabla . \mathrm{q}_{\mathrm{r}}$ of polymer medium regarding the computed temperature at the end of the corresponding iteration step. By this way, the transient heat transfer equation solved for the IR-heating of PE is formulized as:

$$
\rho \cdot C_{p} \frac{\partial \mathrm{T}}{\partial t}=\nabla \cdot(k \nabla \mathrm{T})-\nabla \cdot q_{r}[\mathrm{~T}, x, y, z]
$$

Therefore, the change in the amount of radiative energy absorbed by the PE medium - which is a function of the temperature and the spatial coordinates of the modeled mesh element-, is introduced in the model. The computation time for $\nabla . q_{r}$ was discussed in [13] in terms of number of rays, mesh element and geometrical calculation of modeled rays. In order to reduce the computation cost, the radiative energy that may be absorbed by each modeled 3D mesh element was computed considering the calculated $\beta_{\lambda}(\mathrm{T})$ at 13 different temperature steps and saved before starting the iterative closed-loop temperature field computations. In the model, if a computed temperature in a mesh element is between these 13 steps, the numerical model $\nabla \cdot q_{\mathrm{r}}$ interpolates regarding the $\nabla \cdot \mathrm{q}_{\mathrm{r}}$ of the 2 most close temperature steps. Otherwise the model adopts the $\nabla \cdot \mathrm{q}_{\mathrm{r}}$ of $126^{\circ} \mathrm{C}$, for the mesh element temperatures higher than $126^{\circ} \mathrm{C}$.

\section{Experimental - numerical comparisons for IR-heating of PE plate}

The accuracy of the developed model was analyzed performing an IR-heating test using a rectangular PE plate with dimensions of $78 \times 78 \times 2.2 \mathrm{~mm}$. The plate was heated with a single IR-lamp $(1 \mathrm{~kW})$ at which $100 \%$ of its total power input was utilized. The front surface of the PE plate was positioned $8 \mathrm{~cm}$ away from the IR-lamp.
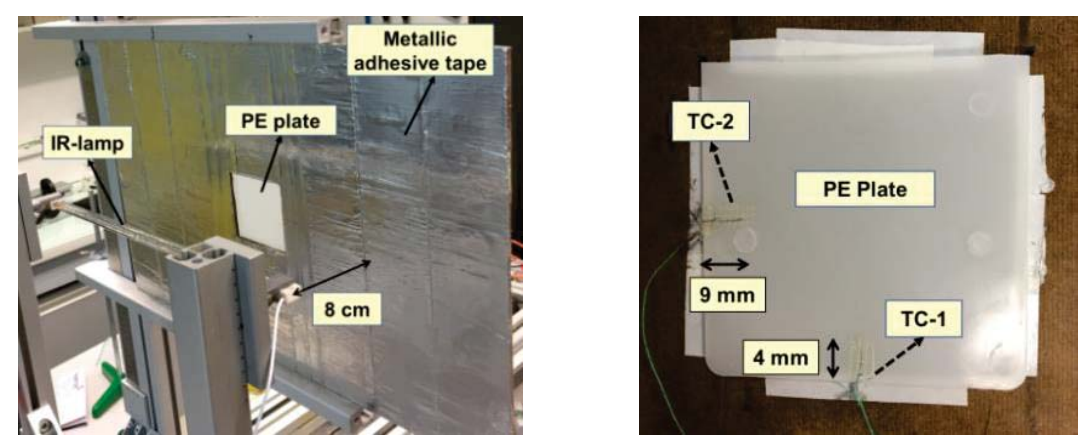

FIGURE 4. Test setup for IR-heating of PE plate (left) and the positions of mounted thermocouples on the back surface (right)

In Fig. 4, the test setup for IR-heating of PE plate is presented at left. As it is seen on that figure, a plate-like barrier is positioned in front the IR-lamp where a small frame $(70 \times 70 \mathrm{~mm})$ is designed for positioning the PE plate. In addition, the front surface of the barrier is covered with metallic adhesive tape in order to avoid heating on the barrier, otherwise conduction heat transfer between the barrier and the PE plate may cause an erroneous temperature measurement. The reason for using a barrier is to perform an IR-thermographic measurement on the back surface of the PE plate, as some of the emitted radiation from IR-lamp may directly reach to the IR-camera and cause noise. For the surface temperature measurements, two thermocouples (TC) were also positioned on the back surface of the plate for comparing their recordings with the IR-thermography. The TC recordings and the IR-heating of the plate were started simultaneously and, it was observed that the temperature on the back surface became steady-state after 600 seconds where also an IR-thermography was taken. Due to the limitations in the IR-thermography of PE that are stressed in [14], the measurement was obtained when the temperature of the plate was at steady-state.

The boundary conditions of the numerical model were built adopting the identical conditions as applied in the experimental test setup. In the model, the plate geometry was meshed with 4232 linear hexagonal elements, and the temperature of each mesh element was calculated as the average temperature of its nodes' temperatures. In the current study, the barrier around the PE plate was not modeled. Based on the findings from the experimental study, heating of the plate up to 600 seconds was simulated using 1500000 rays. The computation of the simulation took around 10 and 55 minutes for the computations of geometrical optics, and the $\nabla . \mathrm{q}_{\mathrm{r}}(\mathrm{T})$, respectively. In Fig. 5, IRthermography of the plate scaled in radiometric values (UI) (a) and, the comparisons between the temperature profiles obtained from the experimental measurements and the numerical studies (b) are presented. The UI values obtained in the IR-thermography was converted into temperature using the methodology in [14]. As displayed in Fig. 5(b), the predicted surface temperature is close (around $3{ }^{\circ} \mathrm{C}$ difference) in the middle zone of the plate regarding the numerically and experimentally defined vertical and horizontal profiles. Conversely, the difference between both the analyses becomes significantly great considering the zones close to the edge of the plate for both 
the horizontal and vertical profiles. This may be attributed to ignorance of heat losses due to conduction between the barrier and PE plate as the barrier around the plate was not modeled. In addition, the convergence studies for the number of modeled rays were not performed at this step which may change the final temperature field predictions.

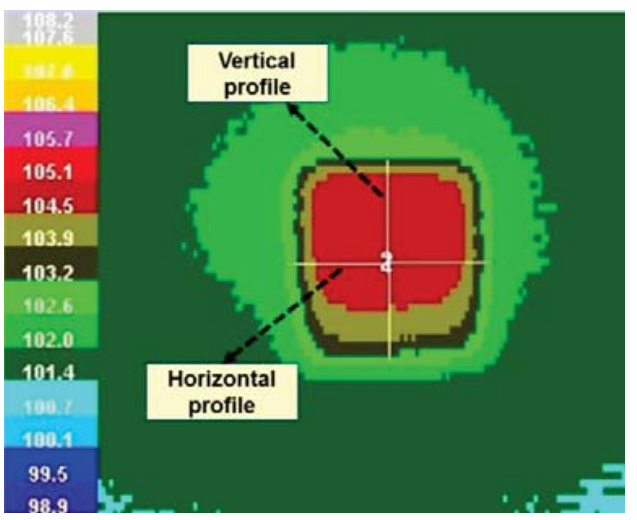

(a)
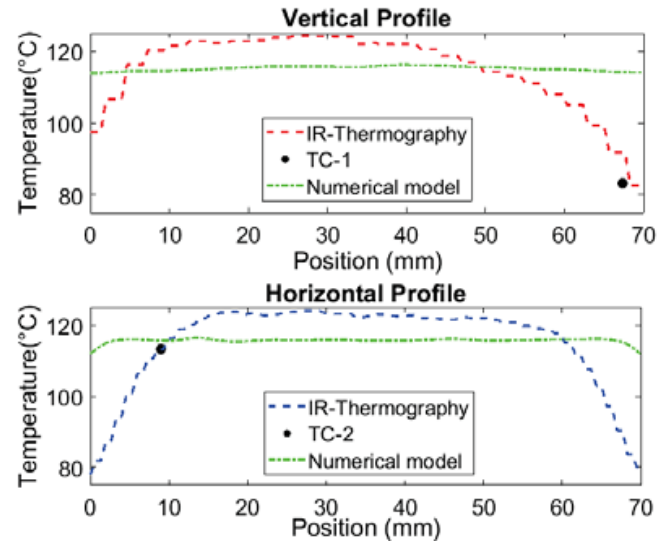

(b)

FIGURE 5. IR-thermography taken at steady-state temperature (a) and the back surface temperature of PE plate obtained via numerical and experimental analyses (b)

\section{CONCLUSIONS}

The model presented in this study proposes a numerical approach to consider the optical scattering and the change in its behavior under heating condition indirectly, without modeling how the light scatters inside of polymer medium. In other words, the effect of optical scattering on the optical characteristics of semi-crystalline PE was taken into account without modeling the spatial distribution of the scattered light intensity which offers computationally cost-effective numerical solutions. The change in the directional-hemispherical optical characteristics of PE under heating conditions were experimentally analyzed and, it was demonstrated especially in the temperature ranges close to the melting range of PE and eventually used as numerical input. In this study, the experimental and numerical analyses showed that the model predicts the temperature closely in the middle zone of the PE plate, whereas there is a remarkable difference at regions close to the edge of the plate considering both the computed and measured surface temperature profiles defined on the normal and alongside to the IR-lamp. This may be attributed to ignorance of potential heat losses due to conduction between the barrier and PE plate as the barrier around the plate was not modeled in this study. In addition, the convergence studies for the number of modeled rays were not performed at this step which may cause to change the final temperature field predictions.

\section{REFERENCES}

1. R. Apetz and M.P.B. Bruggen, J. Am. Ceram. Soc. 86, 480 (2003).

2. M. Ilie, J.-C. Kneip, S. Matteï, A. Nichici, C. Roze, T. Girasole, Opt. Lasers Eng. 45, 405 (2007).

3. E. Berrocal, D.L. Sedarsky, M.E. Paciaroni, I.V. Meglinski, M.A. Linne, Opt.Express 15, 10649 (2007).

4. A. Humphrey, T. Harman, M. Berzins, and P. Smith, in Int. Conf. High Perf. Comput. (2015), pp.212-230.

5. S. Boztepe, R. Gilblas, O. de Almeida, C. Gerlach, Y. Le Maoult, F. Schmidt, Int. J. Mater. Form. (2017).

6. D.V. Tsu, M. Muehle, M. Becker, T. Schuelke, and J. Slagter, Surf. Coat. Technol. 336, 39 (2017).

7. F. Becker and H. Potente, Polym. Eng. Sci. 42, 365 (2002).

8. M. Devrient, X. Da, T. Frick, and M. Schmidt, Phys. Procedia 39, 117 (2012).

9. S. Boztepe, R. Gilblas, O. De Almeida, F. Schmidt, and Y. Le Maoult, in SFT 2017-Congrès Annu. Société Fr. Therm. (2017).

10. S. Boztepe, A. Thiam, O. de Almeida, Y. Le Maoult, F. Schmidt, in $19^{\text {th }}$ Int. ESAFORM Conf. on Mater. Form. (2016).

11. J.R. Howell, M.P. Menguc, and R. Siegel, Thermal Radiation Heat Transfer, $6^{\text {th }}$ Ed. (CRC Press, 2015).

12. A.C.A. Asséko, B. Cosson, F. Schmidt, Y.L. Maoult, E. Lafranche, Infrared Phys.Technol. 72, 293 (2015).

13. B. Cosson, F. Schmidt, Y. Le Maoult, and M. Bordival, Int. J. Mater. Form. 4, 1 (2011)

14. S. Boztepe, R. Gilblas, O. de Almeida, Y. Le Maoult, F. Schmidt, in $20^{\text {th }}$ Int. ESAFORM Conf. on Mater. Form. (2017). 